\title{
Community outbreak of hepatitis A disproportionately affecting men who have sex with men in Toronto, Canada, January 2017-November 2018
}

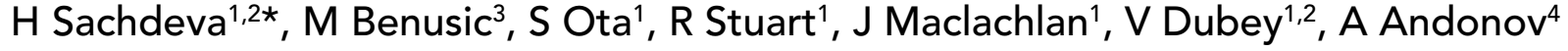

\section{Abstract}

Background: In late 2016 and early 2017, a number of countries began reporting hepatitis A virus (HAV) outbreaks involving person-to-person transmission among men who have sex with men (MSM), people using illicit drugs and homeless or underhoused persons.

Objective: To describe the epidemiology and public health response to an outbreak of HAV disproportionately affecting MSM in Toronto, Canada from January 2017 to November 2018.

Methods: Following an increase in the number of cases of HAV in MSM being reported in other countries, enhanced surveillance was performed for all non-travel-related cases of HAV reported from June 1, 2017 to November 1, 2018, including a retrospective analysis of cases reported from January 2017 to June 2017. Descriptive analysis and viral sequencing were performed to describe person-to-person transmission patterns and target interventions. Control strategies included interventions to promote the uptake of preexposure HAV vaccination, including social media campaigns geared to MSM, messaging to healthcare providers and vaccine clinics.

Results: Based on the outbreak case definitions, 52 confirmed and probable cases of HAV were identified. Over $80 \%$ of outbreak cases were male $(n=43 / 52)$ and, among those for whom data were available, 64\% ( $n=25 / 39)$ reported an MSM exposure. Data on hospitalization was available for 51 cases; $56 \%$ of confirmed cases $(n=23 / 41)$ and $40 \%$ of probable cases $(n=4 / 10)$ required hospitalization. Of the cases with serum samples that had HAV sequencing, $83 \%(n=30 / 36)$ had one of the three strains seen circulating in outbreaks among MSM internationally; 72\% ( $n=26 / 36)$ were VRD_521_2016, which had been detected in recently reported European outbreaks among MSM. Targeted promotion of publicly-funded vaccination using social media platforms popular with MSM and targeted vaccine clinics were developed to promote HAV awareness and vaccine uptake among MSM.

Conclusion: Outbreaks of HAV, attributed to person-to-person transmission of strains of HAV that disproportionately affected MSM and were likely to have been imported from international MSM outbreaks, have now occurred in Canada. Genetic sequencing of HAV, risk factor analysis of cases, monitoring trends of vaccine coverage in high-risk groups and initiation of vaccination campaigns that address barriers to HAV preexposure vaccine coverage in the MSM population may prevent future outbreaks.

Suggested citation: Sachdeva H, Benusic M, Ota S, Stuart R, Maclachlan J, Dubey V, Andonov A. Community outbreak of hepatitis A disproportionately affecting men who have sex with men in Toronto, Canada,

January 2017-November 2018. Can Commun Dis Rep 2019;45(10):262-8.

https://doi.org/10.14745/ccdr.v45i10a03

Keywords: HAV, outbreak, men who have sex with men, vaccine promotion, MSM
This work is licensed under a Creative Commons Attribution 4.0 International License.

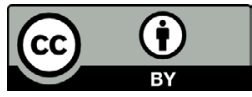

Affiliations

${ }^{1}$ Communicable Disease Control, Toronto Public Health, Toronto, ON

2 Dalla Lana School of Public Health, University of Toronto, Toronto, ON

${ }^{3}$ Public Health \& Preventive Medicine Residency Program, University of Toronto,

Toronto, ON

${ }^{4}$ National Microbiology Laboratory, Winnipeg, MB

*Correspondence: herveen.sachdeva@toronto.ca 


\section{Introduction}

Hepatitis A virus (HAV) infection is endemic in developing countries and is one of the most common vaccine-preventable diseases in travellers (1). Clinical disease results after a 15-50 day incubation period and typically begins with an abrupt onset of fever, nausea and abdominal pain, followed by jaundice (2). While most cases are self-limiting, $25 \%$ of adult cases require hospitalization (1). A case-fatality rate of $2.2 \%$ has been observed among those over 60 years of age (3).

The primary source of HAV is contaminated food or water, but person-to person transmission has also been documented, particularly through sexual anal-oral or digital-anal contact. Men who have sex with men (MSM) have been identified as a higher risk group for person-to-person HAV transmission (4).

In late 2016 and early 2017, a series of HAV outbreaks were reported in countries that had previously had low HAV rates. Specifically, the United States (US), 15 countries in the European Region and Chile documented an increased incidence among MSM (5). In Europe, three co-circulating strains of genotype 1A (VRD_521_2016, V16-25801 and RIVM-HAV16-090) were reported with origins linking to importations from Central America and Asia (4). Also during this time period, US outbreaks were being reported involving individuals reporting illicit drug use or homelessness with genotype lb viral strains (6).

In Ontario, two doses of the HAV vaccine are publicly funded for three groups: MSM; those who use intravenous drugs; and those with pre-existing liver disease (7). Case confirmation of HAV in Ontario requires serology with either compatible symptoms or an epidemiologic link to a confirmed case (8). Toronto Public Health receives, investigates and responds to reports of suspected and confirmed HAV cases. From 2012 to 2016, the average incidence of reported HAV was 32 cases per year (9).

In light of the different high-risk populations being affected by person-to-person HAV transmission internationally $(5,6)$, in June 2017, Toronto Public Health initiated enhanced surveillance of HAV cases, requested hepatitis A genotyping and viral sequencing for all newly reported cases and conducted a retrospective study of the risk factors for cases reported in Toronto since January 2017. Initial analysis in August 2017 showed the number of cases was consistent with the previous five year average for this time period. However, almost $50 \%$ of the cases did not report travel during the incubation period, compared with the previous five year average of $30 \%$. Furthermore, almost $40 \%$ were MSM, compared with the previous five year average of $4 \%$. Locally-acquired HAV cases among MSM continued to be detected in September 2017 and an outbreak was formally declared in October 2017.
The objective of this report is to describe the epidemiology of locally-acquired person-to-person HAV transmission occurring in Toronto, and the public health response to an outbreak of HAV disproportionately affecting MSM from January 2017 to November 2018.

\section{Methods}

\section{Outbreak detection and investigation}

For this investigation, an outbreak case definition was established to include all locally-acquired cases. Confirmed outbreak cases were defined as residents of, or visitors to, Toronto who:

- Met the provincial case definition for a confirmed case of HAV (8)

- Had a report date on or after January 1, 2017

- Had no travel history during their acquisition period

- Had no epidemiological link to a travel-related case

Probable cases were defined as patients having a report date on or after January 1, 2017 and who:

- Met the provincial case definition for a confirmed case of HAV

- Had travelled during the period of acquisition or were epi-linked with a traveller

- Had a risk factor for person-to-person transmission of HAV (homeless or underhoused, use of illicit drugs or MSM).

\section{Case and contact management}

Case management was initiated through a telephone interview guided by a standardized questionnaire from Public Health Ontario (10). As per routine case management procedures, outreach to healthcare providers, shelter staff and harm reduction staff was used to locate harder to reach individuals. Counselling was provided to decrease the risk of transmission to others. Contacts were interviewed to determine eligibility for postexposure prophylaxis. Case and contact information was entered into the Ontario-wide integrated Public Health Information System (iPHIS).

\section{Molecular sequencing}

Hepatitis A virus genotyping and molecular sequencing is not routinely performed in Ontario, but was performed as part of this outbreak investigation. Serum specimens that were sent to the Public Health Ontario Laboratories were forwarded to the National Microbiology Laboratory in Winnipeg, Manitoba for genotyping and molecular sequencing where possible, depending on availability of a serological sample at the diagnosing laboratory. Sequencing results were compared with strains from international outbreaks. 


\section{Analysis}

A line-list of HAV outbreak cases from January 1, 2017 to November 30, 2018 was extracted from iPHIS, and included information on age, gender, address, episode and reported date, risk factors and laboratory results.

Statistical analysis was performed with Stata Statistical Software: Release 15 (College Station, Texas, US: StataCorp LLC). Odds ratios were analyzed using simple logistic regression. Statistical significance was set at alpha $<0.05$.

Home addresses of confirmed outbreak cases were plotted using ArcMap 10.5 (Redlands, California, US: Environmental Systems Research Institute). A heat map was generated using point density and Global Moran's I test for spatial autocorrelation was applied to assess randomness of spatial distribution after applying a fishnet grid for aggregation of points.

In Toronto, some publicly funded vaccines, including HAV vaccines, are ordered directly from Toronto Public Health by healthcare providers. Therefore, the number of publicly funded HAV vaccines ordered by healthcare providers per month was known and were accessed through the Immunization Module of Panorama-the Ontario e-health immunization information system. A t-test was performed on the number of HAV vaccines ordered per month during the outbreak from June 2017 to October 2018 when targeted vaccine promotion efforts were implemented as part of the public health response compared with the number of HAV vaccines ordered per month from January 2012 to May 2017, to help evaluate whether vaccine promotion strategies may have affected vaccine ordering. Vaccines used by public health for contacts for postexposure prophylaxis were not included in this analysis.

\section{Public health response}

Early in the outbreak, all HAV case investigators were trained on enhanced surveillance, including asking about sexual risk factors and sexual orientation. They were also trained on exposures related to illicit drug use.

The following initiatives were undertaken to promote preexposure vaccine uptake among MSM:

1. Alerts were sent to healthcare providers through the Toronto Public Health Communiqué, an e-newsletter with approximately 4,200 subscribers (four updates were sent between June 2017 and June 2018).

2. Three social media campaigns, involving Facebook, Instagram, Twitter and Grindr, were held during the outbreak period (November 2017, January 2018 and one in June preceding the Pride Toronto 2018 festival). Facebook and Grindr were both selected as these platforms allow for targeting an audience very specifically and because users connect socially to one another using these platforms. Grindr, a geosocial networking app oriented towards MSM particularly supported highly targeted vaccine promotion to this population. The campaigns were evaluated and included metrics such as monitoring the number of impressions (how often the messages were viewed), engagement (action taken on the message), web traffic to the Toronto Public Health HAV webpage, and monitoring the relative interest in Ontario web searches of 'hepatitis a' through Google Trends, a campaign evaluation metric that provided insight into online search trends during the outbreak period.

3. A small number of HAV vaccine clinics for MSM were held within the area of Toronto where many lesbian/gay/bisexual/ transgender/queer and others (LGBTQ+) individuals are known to live and where hepatitis cases were clustered (February 2018).

4. Letters were sent to sexual health clinics as well as general healthcare providers who treat sexually transmitted infections (June 2018).

\section{Results}

Figure 1 shows an epidemic curve of outbreak cases by month of onset from January 1, 2017 to November 30, 2018. In total, there were 42 confirmed and 10 probable cases. The outbreak was declared over on November 1, 2018, two months after the last reported case. During the outbreak, Toronto Public Health also received reports of $46 \mathrm{HAV}$ cases that did not meet the outbreak case definition.

Figure 1: Epidemic curve of confirmed and probable outbreak cases of hepatitis A in Toronto, Ontario, by month of onset (January 1, 2017-November 30, 2018)

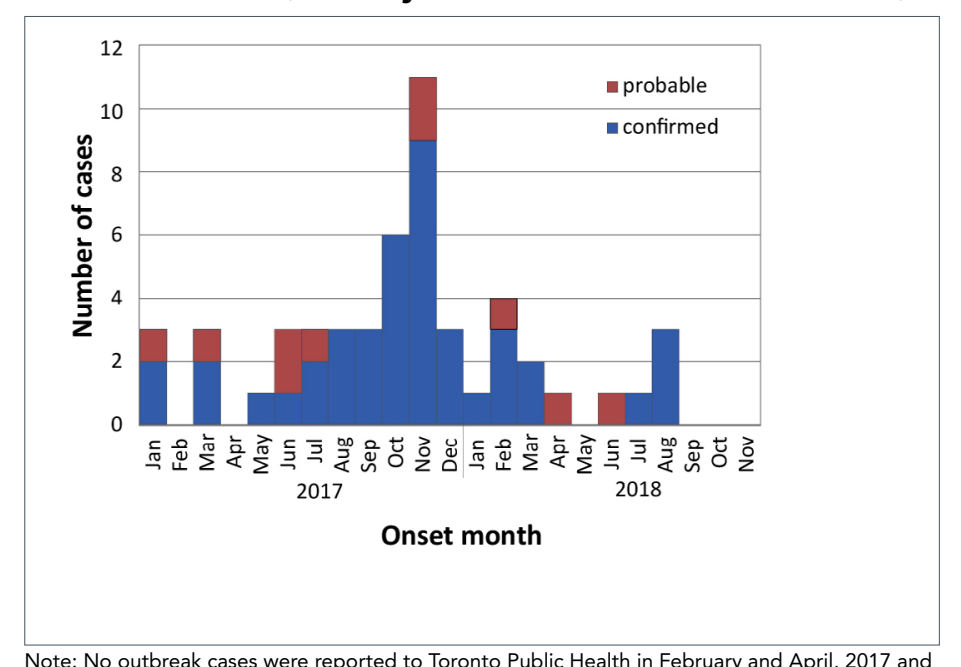

May, September, October and November 2018

Characteristics of confirmed and probable HAV outbreak cases are provided in Table 1. The majority of confirmed outbreak cases were male $(79 \% ; n=33 / 42)$, with a mean age of 38 years. Of the 29 confirmed male cases for which information was available, more than half (59\%; $n=17 / 29$ ) reported an MSM exposure risk factor, in comparison with the previous five year proportion of 
$4 \%$ of HAV cases reporting an MSM exposure risk factor. Nearly half of the confirmed cases (both males and females) reported illicit drug use (49\%; $n=20 / 41)$ and $10 \%(n=4 / 42)$ reported being homeless or underhoused. Of the confirmed cases, the majority of those who reported illicit drug use also reported an MSM exposure risk factor $(65 \% ; n=13 / 20)$. Illicit drug use was often reported as a co-risk factor with MSM and very few cases (4\%) reported illicit drug use in isolation of other risk factors. More than half of the confirmed cases $(56 \% ; n=23 / 41)$ required hospitalization. No outbreak-related risk factors of interest were reported in $43 \%(n=18 / 42)$ of the confirmed cases.

\section{Table 1: Characteristics of hepatitis A virus cases meeting confirmed and probable outbreak case definitions Toronto, Ontario (January 1, 2017-November 30, 2018)}

\begin{tabular}{|c|c|c|c|c|c|c|}
\hline \multirow{3}{*}{ Characteristics $^{\mathrm{a}}$} & \multicolumn{6}{|c|}{ Outbreak cases } \\
\hline & \multicolumn{2}{|c|}{$\begin{array}{c}\text { Confirmed } \\
(n=42)\end{array}$} & \multicolumn{2}{|c|}{$\begin{array}{l}\text { Probable } \\
(n=10)\end{array}$} & \multicolumn{2}{|c|}{$\begin{array}{c}\text { Total } \\
(n=52)\end{array}$} \\
\hline & $\mathbf{n}$ & $\%$ & n & $\%$ & n & $\%$ \\
\hline \multicolumn{7}{|l|}{ Gender } \\
\hline male & $33 / 42$ & 79 & $10 / 10$ & 100 & $43 / 52$ & 83 \\
\hline female & $9 / 42$ & 21 & $0 / 10$ & 0 & $9 / 52$ & 17 \\
\hline \multicolumn{7}{|l|}{ Age } \\
\hline mean age & 38.1 & 38.1 & 39.4 & 39.4 & 38.4 & 38.4 \\
\hline \multicolumn{7}{|l|}{ Risk factors } \\
\hline MSM & $17 / 29$ & 59 & $8 / 10$ & 80 & $25 / 39$ & 64 \\
\hline illicit drug use ${ }^{b}$ & $20 / 41$ & 49 & $3 / 9$ & 33 & $23 / 50$ & 46 \\
\hline $\begin{array}{l}\text { illicit drug use }{ }^{b} \text { with no } \\
\text { other risk factors }\end{array}$ & $2 / 36$ & 6 & $0 / 9$ & 0 & $2 / 45$ & 4 \\
\hline cannabis use & $9 / 40$ & 23 & $3 / 9$ & 33 & $12 / 49$ & 24 \\
\hline travel & $0 / 38$ & 0 & $10 / 10$ & 100 & $10 / 48$ & 21 \\
\hline homeless/underhoused & $4 / 42$ & 10 & $0 / 10$ & 0 & $4 / 52$ & 8 \\
\hline none reported & $18 / 42$ & 43 & $0 / 10$ & 0 & $18 / 52$ & 35 \\
\hline \multicolumn{7}{|l|}{ Co-infections } \\
\hline HIV co-infection & $6 / 40$ & 15 & $3 / 10$ & 30 & $9 / 50$ & 18 \\
\hline previous/current STI & $13 / 40$ & 33 & $5 / 10$ & 50 & $18 / 50$ & 36 \\
\hline \multicolumn{7}{|l|}{ Morbidity } \\
\hline hospitalization & $23 / 41$ & 56 & $4 / 10$ & 40 & $27 / 51$ & 53 \\
\hline \multicolumn{7}{|l|}{ Strain } \\
\hline VRD_521_2016 & $26 / 32$ & 81 & $0 / 4$ & 0 & $26 / 36$ & 72 \\
\hline RIVM HAV16-090 & $1 / 32$ & 3 & $2 / 4$ & 50 & $3 / 36$ & 8 \\
\hline V16-25801 & $0 / 32$ & 0 & $1 / 4$ & 25 & $1 / 36$ & 3 \\
\hline non-outbreak strain & $5 / 32$ & 16 & $1 / 4$ & 25 & $6 / 36$ & 17 \\
\hline
\end{tabular}

Abbreviations: MSM, men who have sex with men; STI, sexually-transmitted infections

Denominators reflect counts of cases where information on each characteristic was collected ${ }^{b}$ Reported illicit drugs included cannabis, G liquid, 3,4-methylenedioxymethamphetamine (MDMA, ecstasy), opioids/fentanyl, non-injection cocaine, crystal methamphetamine, heroin and crack
Sequencing results were received for 36 cases (confirmed and probable). Of these, $83 \%(n=30 / 36)$ had one of the three strains seen circulating in outbreaks among MSM internationally. The majority $(72 \% ; n=26 / 36)$ were the VRD_521_2016 strain. This outbreak strain was noted as the most common strain in the MSM outbreaks reported in the European Union (4) and was also reported in South American outbreaks affecting MSM and in New York City $(11,12)$. An outbreak strain was significantly more likely to be sequenced among confirmed cases where at least one risk factor was reported as compared with those where no risk factor of interest was reported (odds ratio: $8.14 ; 95 \%$ confidence interval: 1.32-50.3).

Home addresses were obtained and mapped for 38 confirmed outbreak cases. Global Moran's I test for spatial autocorrelation showed that spatial distribution was statistically not random (Moran's Index: 0.15, p-value $<0.001$ ) and there was relative clustering observed in one of the areas in downtown Toronto popular with MSM and LGBTQ+ communities.

Evaluation of the social media campaigns showed that messaging timed with the Pride Toronto 2018 festival may have had the most impact in terms of number of views and engagement with the campaign. Figure 2 shows a graph of the Google search term for 'hepatitis a' for Ontario over time relative to the highest point of interest, which coincided with the Pride Toronto festival in June 2018.

Figure 2: Number of Google searches on 'hepatitis $A$ ' in Ontario (August 2017-November 2018)

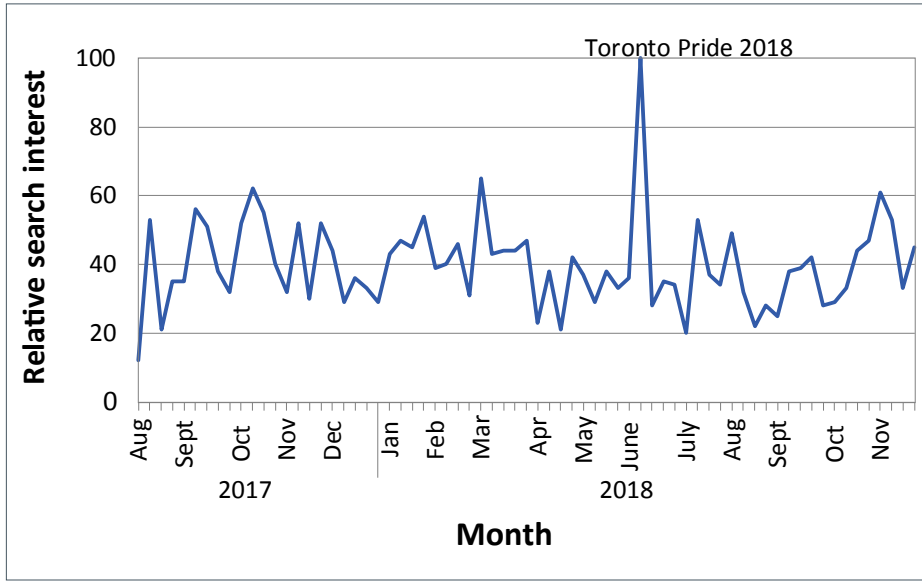

Notes: Y-axis reflects search interest over time ( $\mathrm{x}$ axis). The highest point on the chart coincided with Toronto Pride in June 2018

Orders for HAV vaccine increased during the peak of the outbreak (June 2017 to November 2018) compared with before the outbreak. The monthly mean number of HAV vaccines ordered from June 2017 to October 2018 was 348, compared with a monthly mean of 257 from January 2012 to May 2017 $(t=4.72, p<0.001)$. A total of 105 vaccines were given at the six MSM preexposure clinics that were held by Toronto Public Health in early 2018. 


\section{Discussion}

To our knowledge, this is the first published report of a sustained HAV outbreak disproportionately affecting an MSM population in Canada in over twenty years. Several outbreaks from the 1990s have been summarized (13). Then in 2016 and 2017, multiple HAV outbreaks affecting MSM were reported internationally $(4,5,11,12)$. In the $2017-2018$ outbreak we described, clustering was observed in an area of Toronto where many people live who identify as being part of the MSM and LGBTQ+ communities. Viral sequencing of the strains of this outbreak matched those of the international outbreaks. Social media and other outreach strategies used in similar outbreaks (e.g. New York City, US) were used in the Toronto outbreak to develop targeted vaccine promotion.

All three HAV strains originating in recent international MSM outbreaks (5) were detected in this outbreak, with VRD_521_2016 being the most common. VRD_521_2016 was also the first strain noted to be circulating in the European Union MSM outbreaks (5). Based on the epidemiological curve, sequencing information and travel information collected from cases reporting MSM exposure, it is possible that importation of the strains circulating outside of Canada occurred prior to and during Pride Toronto 2017. This suggests importation of these strains into Toronto in June 2017 and subsequent local transmission through to mid-2018. As other recent outbreak reports in low endemic areas have concluded, a combination of international travel and sexual networks can sustain a large outbreak of HAV within a susceptible population (4).

In Ontario, MSM can access the publicly funded HAV vaccine for preexposure prophylaxis. This program has been in place since 1997; however, this recent outbreak demonstrates that immunity levels among MSM were not sufficient to avert an outbreak. Currently, to obtain the publicly funded HAV vaccine, healthcare providers need to document MSM, drug use or other risk factors, and to offer safer sex counselling. The need for disclosure of these risk factors may reduce the effectiveness of the high-risk HAV vaccination program and may be a barrier to maintaining sufficiently high preexposure vaccination coverage. Prior modelling studies have estimated that immunity levels of $70 \%$ or more would be needed among MSM to prevent an outbreak (14). Illicit drug use among cases was monitored, and there was significant overlap in cases who reported an MSM exposure and a drug use exposure. This finding may be related to prior research among MSM that has shown a relationship between substance use and increased sexual risk behaviors (15).

Alerts to healthcare providers, social media and traditional communication campaigns were used to increase awareness of the outbreak and promote vaccination and these were followed by a significant increase in HAV vaccine orders by Toronto healthcare providers. Only four new cases were reported after the Pride Toronto 2018 campaign, and the outbreak was declared over in November 2018. The use of social media campaigns appeared to be an effective strategy to raise awareness of HAV among MSM in Toronto.

\section{Limitations}

This analysis, based only on HAV cases that were reported, likely underestimates the true extent of this outbreak. The hospitalization rate observed in this outbreak was higher than typically quoted for this disease; $56 \%$ of confirmed cases required hospitalization during this outbreak. However, this is similar to another report that hypothesised the higher hospitalization rate may be due to increased case ascertainment (4).

For 18 confirmed cases, a risk factor of interest for locally-acquired person-to-person transmission could not be ascertained; their epidemiologic link to the outbreak remains unknown. These cases were significantly less likely to have an outbreak viral sequence and, therefore, may be unrelated to the outbreak. Cases with the outbreak viral sequence may represent local transmission into the general population, as reported in other outbreaks $(16,17)$. Or, these cases may have been misclassified due to reluctance of cases to disclose sexual activities, orientation or illicit drug use. As the outbreak progressed, Toronto Public Health staff changed their interview practices to more specifically ascertain risk factor information for local HAV cases. Therefore, comparisons to prior years should be interpreted with caution.

An online vaccine ordering platform was introduced during the outbreak time period and may have contributed to higher vaccine orders. Further, the data pulled from Panorama did not enable us to separate out vaccine orders for MSM compared with other high-risk groups. The extent of the baseline vaccination coverage of MSM in Toronto is unknown; therefore, the degree to which this population is protected is also unknown.

\section{Conclusion}

Outbreaks of HAV, caused by person-to-person transmission of strains of HAV that disproportionately affect MSM have now occurred in Canada. These strains were likely to have been imported from international MSM outbreaks. At-risk populations were identified using a combination of HAV sequencing results and descriptive analysis of risk factor information-facilitating targeted vaccine promotion. The use of targeted social media campaigns appears to be an effective strategy to promote HAV awareness and vaccine uptake among MSM during the outbreak period. Periodic assessment of vaccine coverage rates in MSM and further study on how best to ensure they are maintained at high levels may help to mitigate future outbreaks. 


\section{Authors' statement}

HS oversaw the outbreak management and the writing of the manuscript $\mathrm{MB}$ conceived the first draft of this manuscript

$\mathrm{SO}$ and $\mathrm{MB}$ analyzed the epidemiological data

$J M$ oversaw and evaluated the social media campaigns

$V D, J M, R S$, and SO were involved in the outbreak management

AA provided viral sequencing and interpretation

All authors were involved in the editing and revisions of the manuscript

\section{Conflict of interest}

None.

\section{Acknowledgements}

The Toronto Public Health Hepatitis A outbreak management group (including D Hayden, K Beckermann, K Bradley and A Summers) facilitated the data collection and response measures.

M Finkelstein and CC Hui (the latter on behalf of the Toronto Public Health men who have sex with men workgroup) helped edit the manuscript.

Staff and management in multiple program areas at Toronto Public Health contributed to the control of the outbreak. The authors also wish to acknowledge the support of Public Health Ontario Laboratory, the National Microbiology Laboratory and the New York City Department of Health and Mental Hygiene.

\section{References}

1. Public Health Agency of Canada Canadian Immunization Guide. Part 4 - Active Vaccines: Hepatitis A Vaccine. PHAC; Ottawa (ON); 2018 (Accessed 2018-12-06). www.canada. ca/en/public-health/services/publications/healthy-living/ canadian-immunization-guide-part-4-active-vaccines/page6-hepatitis-a-vaccine.html\#p4c5a2

2. Centers for Disease Control and Prevention. Chapter 9: Hepatitis A. In: Epidemiology and prevention of Vaccine Preventable Diseases (The Pink Book, 13th Edition). CDC; 2015 (Accessed 2018-12-02). www.cdc.gov/vaccines/pubs/ pinkbook/hepa.html

3. National Advisory Committee on Immunization. An Advisory Committee Statement (ACS). Update on the Recommended use of Hepatitis A Vaccine. PHAC; 2016 (Accessed 201812-02). www.canada.ca/content/dam/hc-sc/documents/ services/publications/healthy-living/hepatitis-a-vaccine-updat e-recommended-use-2016-eng.pdf
4. Ndumbi P, Freidl GS, Williams CJ, Mårdh O, Varela C, Avellón A, Friesema I, Vennema H, Beebeejaun K, Ngui SL, Edelstein M, Smith-Palmer A, Murphy N, Dean J, Faber M, Wenzel J, Kontio M, Müller L, Midgley SE, Sundqvist L, Ederth JL, Roque-Afonso AM, Couturier E, Klamer S, Rebolledo J, Suin V, Aberle SW, Schmid D, De Sousa R, Augusto GF, Alfonsi V, Del Manso M, Ciccaglione AR, Mellou K, Hadjichristodoulou C, Donachie A, Borg ML, Sočan M, Poljak M, Severi E; Members Of The European Hepatitis A Outbreak Investigation Team. Hepatitis A outbreak disproportionately affecting men who have sex with men (MSM) in the European Union and European Economic Area, June 2016 to May 2017. Euro Surveill 2018 Aug;23(33):1700641. DOI PubMed

5. World Health Organization. Hepatitis A outbreaks mostly affecting men who have sex with men - European Region and the Americas. WHO; 2017 (Accessed 2018-12-06). www.who.int/csr/don/07-june-2017-hepatitis-a/en/

6. Foster M, Ramachandran S, Myatt K, Donovan D, Bohm S, Fiedler J, Barbeau B, Collins J, Thoroughman D, McDonald E, Ballard J, Eason J, Jorgensen C. Hepatitis A Virus Outbreaks Associated with Drug Use and Homelessness - California, Kentucky, Michigan, and Utah, 2017. MMWR Morb Mortal Wkly Rep 2018 Nov;67(43):1208-10. DOI PubMed

7. Ontario Ministry of Health and Long-Term Care. Publicly Funded Immunization Schedules for Ontario - December 2016. OMHLTC; 2016 (Accessed 2018-12-02).

www.health.gov.on.ca/en/pro/programs/immunization/docs/ immunization_schedule.pdf

8. Ontario Ministry of Health and Long-Term Care. Infectious Disease Protocol. Appendix B: Provincial Case Definitions for Reportable Diseases. Disease: Hepatitis A. OMHLTC; 2017 (Accessed 2018-12-02).

www.health.gov.on.ca/en/pro/programs/publichealth/oph_ standards/docs/hep_a_cd.pdf

9. Toronto Public Health Communicable Disease Surveillance Unit. Communicable Diseases in Toronto - 2017. www.toronto.ca/community-people/health-wellness-care/ health-inspections-monitoring/communicable-diseasestoronto/

10. Public Health Ontario. Ontario Investigation Tools (Standardized Questionnaires). PHO; 2018 (Accessed 2018-12-02). pp. 1-2. www.publichealthontario. ca/en/BrowseByTopic/InfectiousDiseases/Pages/ Standardized-Questionnaires.aspx

11. Latash J, Dorsinville M, Del Rosso P, Antwi M, Reddy V, Waechter H, Lawler J, Boss H, Kurpiel P, Backenson PB, Gonzalez C, Rowe S, Poissant T, Lin Y, Xia GL, Balter S. Notes from the Field: Increase in Reported Hepatitis A Infections Among Men Who Have Sex with Men - New York City, January-August 2017. MMWR Morb Mortal Wkly Rep 2017 Sep;66(37):999-1000. DOI PubMed 
12. Mariojoules J, Castro G, Pisano MB, Barbero P, Fantilli A, Borda M, Canna F, Barbás G, Ré V. Hepatitis A outbreak affecting men who have sex with men (MSM) in central Argentina, occurred in July 2017-April 2018, later than the European outbreak. J Clin Virol 2019 Aug;117:49-53. DOI PubMed

13. Uhlmann S, Buxton JA. A provincial and territorial review of hepatitis $A$ in men who have sex with men. Can Commun Dis Rep. 2007 Oct 1;33(11):1-11. https://www.canada.ca/en/public-health/services/ reports-publications/canada-communicable-disease-reportccdr/monthly-issue/2007-33/canada-communicabl e-disease-report.html

14. Regan DG, Wood JG, Benevent C, Ali H, Smith LW, Robertson PW, Ferson MJ, Fairley CK, Donovan B, Law MG. Estimating the critical immunity threshold for preventing hepatitis A outbreaks in men who have sex with men. Epidemiol Infect 2016 May;144(7):1528-37. DOI PubMed
15. Bourne A, Weatherburn P. Substance use among men who have sex with men: patterns, motivations, impacts and intervention development need. Sex Transm Infect 2017 Aug;93(5):342-6. DOI PubMed

16. Friesema IH, Sonder GJ, Petrignani MW, Meiberg AE, van Rijckevorsel GG, Ruijs WL, Vennema H. Spillover of a hepatitis A outbreak among men who have sex with men (MSM) to the general population, the Netherlands, 2017. Euro Surveill 2018 Jun;23(23):1800265. DOI PubMed

17. Lanini S, Minosse C, Vairo F, Garbuglia A, Di Bari V, Agresta A, Rezza G, Puro V, Pendenza A, Loffredo MR, Scognamiglio P, Zumla A, Panella V, Ippolito G, Capobianchi MR; Gruppo Laziale Sorveglianza Epatiti Virali (GLaSEV). A large ongoing outbreak of hepatitis A predominantly affecting young males in Lazio, Italy; August 2016 - March 2017. PLoS One 2017 Nov;12(11):e0185428-14. DOI PubMed

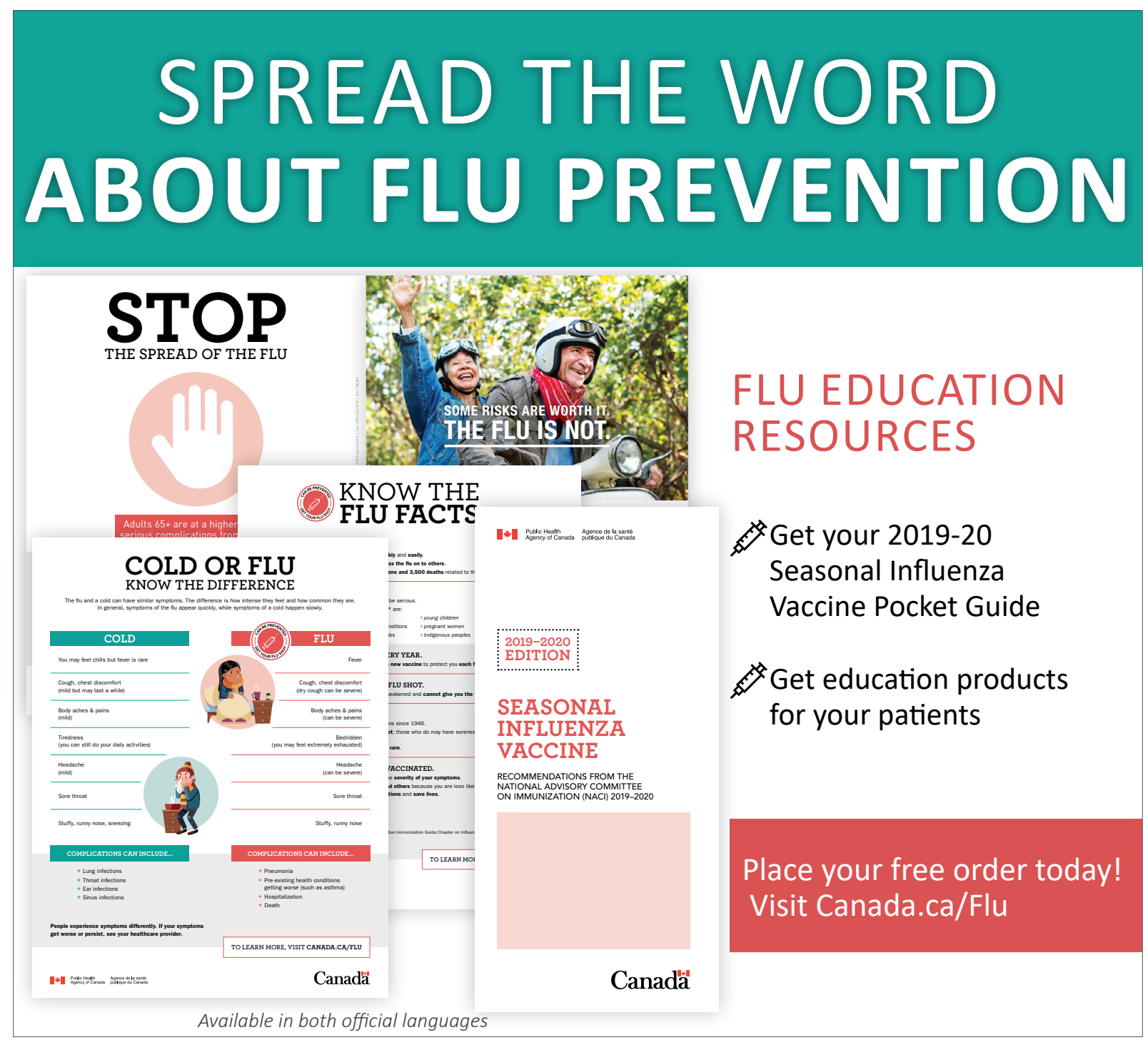

